\title{
Aplicação de técnicas de análise exploratória no monitoramento da qualidade da água do rio Cuiá, João Pessoa-PB
}

\section{Application of exploratory analysis techniques in monitoring the water quality of the Cuia river, João Pessoa-PB}

\author{
Diego Gomes Sousa' $(*)$ \\ Debora Santos Marques ${ }^{2}$ \\ Vilhena Silva Santos ${ }^{3}$ \\ Antonio Cícero Sousa ${ }^{4}$ \\ Gesivaldo Alves Figueiredo ${ }^{5}$
}

\section{Resumo}

A manutenção da qualidade da água de rios é de suma importância para a garantia dos usos preponderantes previsto na resolução Conama 357/2005, bem como a conservação do meio-ambiente. O uso de ferramentas estatísticas multivariadas se mostra relevante, assim permitindo uma visão mais global dos fenômenos ambientais envolvidos, as quais permitem que se extraiam informações que possibilitem uma compreensão da relação entre os parâmetros estudados. O presente trabalho tem como objetivo avaliar as variáveis que afetam a qualidade da água do rio Cuiá, bem como sua relação com os fatores ambientais e atividades antrópicas, empregando análise hierárquica (HCA) e análise de componente principal (PCA). Foram coletadas da bacia do rio Cuiá, vinte e quatro amostras de água de quatro pontos em três campanhas amostrais, realizados nos meses de agosto/2017, novembro/2017 e maio/2018, a fim de associar os resultados das análises físicas, químicas e microbiológicas com as atividades humanas e climáticas. A análise de HCA mostrou que ocorre a formação de dois grupos de variáveis, com a presença de algumas variáveis adicionais para um dos conjuntos. A análise de PCA evidenciou que ocorre uma segregação ao efetuar uma análise temporal e espacial. A aplicação da análise exploratória multivariada mostrou-se relevante na verificação de

1 Mes.; ECOLOGIA E TECNOLOGIA AMBIENTAL; Universidade Federal de Alfenas, UNIFAL/MG, Brasil; Analista de Projetos Pleno na empresa Integratio - Mediação Social e Sustentabilidade Ltda; Endereço: Av. Getúlio Vargas, 1671 - 7o Andar - Savassi, Belo Horizonte - MG, CEP:30112-024; E-mail: diegogomees28@gmail.com (*) Autor para correspondências

$2 \quad$ E-mail: debrinhha.janine@gmail.com

3 Graduanda; Licenciatura em Química; Instituto Federal de Educação, Ciência e Tecnologia da Paraíba, IFPB, Brasil; E-mail: vilhena.leticia@gmail.com

4 Dr.; Química; Universidade Federal da Paraíba, UFPB, Brasil; Professor desde setembro de 1993 do Instituto Federal de Educação, Ciência e Tecnológica da Paraíba (IFPB); Endereço: Instituto Federal de Educação, Ciência e Tecnologia da Paraíba, IFPB - Campus João Pessoa, Unidade Sede de João Pessoa. Rua primeiro de maio 720 (Área de Tecnologia Ambiental / Licenciatura em quimica). Jaguaribe, CEP: 58015-430 - Joao Pessoa, PB - Brasil; E-mail: antoniocicero@ yahoo.com

5 Dr.; Engenharia Agrícola; Universidade Federal de Campina Grande, UFCG, Brasil; Professor efetivo do Instituto Federal de Educação, Ciência e Tecnologia da Paraíba (IFPB), campus João Pessoa; Endereço: Instituto Federal de Educação, Ciência e Tecnologia da Paraíba, Coordenação do Curso de Licenciatura em Química. Av.: 1º de Maio, 720, Jaguaribe. CEP: 58015430 - João Pessoa, PB - Brasil; E-mail: gesivaldojesus@hotmail.com

\begin{tabular}{llllll}
\hline Ambiência & Guarapuava (PR) v.l5 n.l & p. I3I - I45 Jan/Abr 2019 & ISSN I808 - 025I
\end{tabular}


padrões comportamentais, tanto da análise temporal quanto espacial. Os resultados de PCA indicam que as amostras da zona de mistura apresentam maior contaminação por Escherichia coli (EC) e coliformes totais (CT) do que as coletadas a montante e a jusante do ponto de lançamento da estação de tratamento de esgoto doméstico de Mangabeira (ETE-Mangabeira). Espera-se que esses resultados possam subsidiar a tomada de decisão dos gestores da bacia hidrográfica do rio Cuiá frente às ameaças de contaminação desse ecossistema.

Palavras-chave: Qualidade da água.PCA.Água superficial. Análise exploratória.

\section{Abstract}

The maintenance of the quality of river water is of paramount importance for the guarantee of the prevailing uses envisaged in Conama Resolution 357/2005, as well as the conservation of the environment. The use of multivariate statistical tools is relevant, thus allowing a more global view of the environmental phenomena involved, which allow the extraction of information that allows an understanding of the relationship between the studied parameters. The objective of this study was to evaluate the variables that affect Cuia river water quality, as well as its relationship with environmental factors and anthropic activities, using hierarchical analysis (HCA) and principal component analysis (PCA). Twenty - four water samples of four points were collected from the Cuia river basin in three sampling campaigns, conducted in August / 2017, November / 2017 and May / 2018, in order to associate the results of the physical, chemical and with human and climatic activities. Analysis of HCA showed that two groups of variables occur, with the presence of some additional variables for one of the sets. The PCA analysis showed that segregation occurs when performing a temporal and spatial analysis. The application of multivariate exploratory analysis proved to be relevant in the verification of behavioral patterns, both temporal and spatial analysis. The PCA results indicate that the samples from the mixing zone show higher contamination by Escherichia coli (EC) and total coliforms (TC) than those collected upstream and downstream of the domestic wastewater treatment plant (ETE-Mangabeira) launch point. It is hoped that these results could support the decision-making of managers of the Cuia river basin in face of the threats of contamination of this ecosystem.

Keywords: Water quality. PCA. Surface water. Exploratory analysis

\section{Introdução}

A preocupação com a escassez hídrica exige reflexões sobre a utilização dos recursos da natureza em todos os países, industrializados e em desenvolvimento, no âmbito das esferas ambiental, social, política e econômica. É notório que a água do planeta está se esgotando gradativamente e que, além da poluição dos rios e dos mananciais, o consumo indiscriminado, de forma irresponsável e sem fundamentação sustentável no desenvolvimento econômico é um fator relevante que contribui para sua redução (DETONI, 2008).

A Organização das Nações Unidas (ONU) alerta que a escassez de água afetará cerca de dois terços da população mundial em 2050, em consequência do uso excessivo dos recursos hídricos para a produção de alimentos e para a agricultura (FAO, 2015). 
Estudos realizados por Telles at al. (2013) mostram que a água é afetada por diversos fatores, dentre eles, o desenvolvimento urbano, industrial e rural, que está diretamente ligado à oferta de recursos hídricos. Ainda nesse estudo, os autores revelam que a sua disponibilidade envolve o fator quantidade, bem como o fator qualidade, que interfere diretamente no desenvolvimento geral da saúde dos ecossistemas e das comunidades humanas servidas. Contudo, essa escassez é ocasionada pelos mais diversificados usos, pelo mau gerenciamento, pelas péssimas condições ambientais e pelo modelo de sistema produtivo vigente, que tem deixado marcas de degradação, a exemplo da poluição e da contaminação de um modo geral.

Os rios são cursos d'águas, usualmente de água doce considerados sistemas lóticos complexos caracterizados como escoadouros naturais das áreas de drenagens adjacentes, que formam as bacias hidrográficas. A complexidade desses sistemas deve-se ao uso do solo, geologia, tamanho e formas das bacias de drenagem, além das condições climáticas locais (TOLEDO; NICOLLELA, 2002).

Entende-se como qualidade da água de um rio, um conjunto de todas as características físicas, químicas e biológicas que ela apresenta a qual pode ser afetada em qualquer ponto por várias interferências, incluindo litologia da bacia, condições climáticas e interferências antropogênicas (LEMOS, 2011). Neste sentido, tanto os processos naturais, a saber, clima, precipitação, erosão, intemperismo, geologia, tipo de solo, quanto os antropogênicos, tais como atividades urbanas, industriais e agrícolas, bem como o aumento da exploração dos recursos hídricos, podem determinar a qualidade da água de uma determinada região (MENDIGUCHÍA et al, 2004; SINGH et al., 2004; VEGA et al., 1998).

As águas superficiais, a exemplo dos rios, são muito suscetíveis à poluição devido ao seu fácil acesso, uma vez que desempenham um papel importante na assimilação ou transporte de águas residuárias municipais e industriais e no escoamento de terras agrícolas (SINGH et al., 2004; VIDAL et al., 2000).

O lançamento de efluentes domésticos e industriais nos corpos d'águas constitui uma constante fonte de poluição, enquanto o escoamento superficial é um fenômeno sazonal, em grande parte afetada pelo clima na bacia (SUNDARY et al., 2005; VEGA et al., 1998). Os impactos ambientais provenientes das águas residuárias municipais e da descarga de efluentes industriais em águas receptoras são numerosos e a entrada de contaminantes pode afetar sua qualidade ao ponto de torná-la inadequada para o consumo humano, recreação, indústria e agricultura, dentre outras (MENDIGUCHÍA et al., 2004).

Segundo Hidalgo (1992), qualquer variação das características físicas, químicas ou biológicas dos recursos naturais, a saber, solo, vegetação, ar, água e fauna, causada por qualquer forma de poluição resultante de atividades humanas afetam diretamente a bacia hidrográfica, gerando condições nocivas à saúde da população que depende da sua drenagem. A variação de tais características reflete a qualidade da água no qual estão associadas a uma série de processos que ocorrem no corpo hídrico e em sua bacia de drenagem.

Segundo Calijuri (1999), os reservatórios de águas do Brasil têm apresentado, em geral, grandes alterações nos seus estados tróficos, devido ao uso e ocupação das bacias hidrográficas. Para evitar tais alterações faz-se necessário estabelecer um manejo integrado dos reservatórios no qual é fundamental o conhecimento aprofundado desses sistemas. Para tanto é imprescindível a implementação de um monitoramento da qualidade da água, pois, além de registrar as variações espaço-temporais, relaciona os diversos mecanismos do sistema hídrico com toda a sua bacia de drenagem (JONG et al., 1995). 
O Brasil contempla, no âmbito da legislação ambiental, uma série de leis que estão em vigência no país, dentre essas, a Lei Federal no 9.433/97 que estabelece a bacia hidrográfica como "unidade territorial para aplicação da Política Nacional de Recursos Hídricos (PNRH), e atuação do Sistema Nacional de Gerenciamento de Recursos Hídricos", no qual se iniciou uma fase de conscientização sobre a preservação e manutenção dos diferentes recursos hídricos presentes no território nacional (BRASIL, 1997); a Resolução CONAMA 357/2005 que dispõe sobre a classificação dos corpos de água e diretrizes ambientais para o seu enquadramento, a Resolução CONAMA 430/2011 que estabelece as condições e padrões de lançamento de efluentes. Tais resoluções criaram ferramentas para auxiliar a Agência Nacional das Águas (ANA) no desenvolvimento de suas atividades, pois elas estabelecem os critérios e parâmetros químicos, físicos e microbiológicos necessários para efetuar a classificação e enquadramento dos diferentes mananciais (BRASIL, 2005 e 2011).

Segundo a resolução CONAMA 357/2005, os níveis de exigência da qualidade da água são apresentados de acordo como cada finalidade, a saber, uso na indústria, na irrigação, dessedentação de animais, dentre outro e o abastecimento humano que requer grande atenção (BRASIL, 2005).

A luz de Sousa (2007), a dinâmica dos sistemas hídricos é multifatorial e de complexa compreensão, tendo em vista os diversos fatores que podem influenciar, dentre esses, podemos citar a interação entre constituintes do meio aquático e os compartimentos ambientais, a saber, hidrosfera, litosfera e atmosfera. Tais interações dificultam o estudo e a interpretação dos sistemas envolvidos. Nesse sentido, para uma melhor compreensão, é imprescindível o uso de ferramentas estatísticas multivariadas, uma vez que estas apresentam uma visão mais global dos fenômenos ambientais, os quais, na maioria das vezes, não são percebidos por ferramentas convencionais.

Segundo Hair Junior et al. (2005), a análise fatorial é uma técnica de análise multivariada e de interdependência, na qual todas as variáveis são consideradas simultaneamente, sendo cada uma relacionada com todas as outras e ainda empregando o conceito da variável estatística, bem como a composição linear de variáveis. Nessa análise, as variáveis estatísticas (fatores) são formadas para maximizar seu poder de explicação de todo conjunto de variáveis e não para prever apenas uma variável dependente.

Dentre as análises fatoriais, podemos citar a análise de componentes principais (PCA) que é um método de análise multivariada utilizado para projetar os objetos (pontos) na direção do espaço dimensional das vaiáveis que contenham a maior variância possível em um espaço de baixa dimensão, normalmente duas ou três. Isso é realizado por meio do cálculo de componentes principais obtidos fazendo-se combinações lineares das variáveis originais (SOUSA, 2007).

A análise de componente principal é um método exploratório porque auxilia na elaboração de hipóteses gerais a partir dos dados coletados, confrontando com estudos direcionados, nos quais hipóteses são testadas a priori. Essa técnica também é capaz de separar as informações relevantes das redundantes e aleatórias. Em uma PCA o agrupamento das amostras define a estrutura do conjunto de dados através dos gráficos dos escores (amostras) e pesos (variáveis), cujos eixos são os componentes principais (PCs) nos quais os dados são projetados. Tendo em vista que uma PC é ortogonal a outra, é possível examinar as relações entre amostras e variáveis através dos gráficos dos escores e dos pesos, assim o estudo do conjunto dos escores e dos pesos possibilita estimar a influência de cada variável em cada amostra (FERREIRA, 1999; HASWELL, 1998; SENA, 2000). 
A análise hierárquica de agrupamentos (HCA) é uma técnica multivariada cuja finalidade primária é agregar objetos com base nas características que eles possuem. Os resultados são apresentados na forma de dendrogramas, os quais agrupam amostras ou variáveis em função da similaridade. Essa similaridade pode ser medidas por três métodos, a saber, medidas correlacionais, medidas de distâncias e medidas de associação. Estudos mostram que a distância euclidiana e a técnica de conexão baseada na distância do vizinho mais próximo são as metodologias mais utilizadas para o cálculo da similaridade (BUSSAD, 1990; HAIR, 2005).

Enio et al. (2007) empregou a técnica de PCA com a finalidade de identificar os parâmetros físicos e químicos mais relevantes na variabilidade da qualidade da água no trecho perenizado do rio Jaibaras no estado do Ceará. Os resultados de PCA permitiu verificar o efeito dos parâmetros químicos na variação espacial da qualidade das águas superficiais no trecho perenizado do rio Jaibaras. Os estudos mostraram qua a qualidade da água recebe maior influência da ação antrópica (dejetos dos balneários, fertilizantes nitrogenados e esgotos domésticos).

Desta forma, o uso de análise de agrupamento hierárquico (HCA) e de componente principal (PCA) torna-se ferramenta relevante, tendo em vista que as variáveis são originárias de processos em que diversas características devem ser observadas simultaneamente (SOUSA, 2007).

$\mathrm{O}$ presente trabalho tem como objetivo o emprego de ferramentas multivariadas no monitoramento de águas superficiais oriundas da Bacia Hidrográfica do Rio cuiá,João Pessoa-PB.

\section{Material e Métodos}

\section{Caracterização da área de estudo}

$\mathrm{O}$ rio Cuiá nasce nas encostas dos rebordos dos tabuleiros sedimentares costeiros no setor sul da capital do estado da Paraíba, cortando vinte e um bairros da malha urbana, contemplando por completo os bairros de Água Fria, Anatólia, Boa Esperança, Cidade dos Colibris, Cuiá, José Américo, Valentina e Paratibe e parcialmente os bairros dos Bancários, Barra do Gramame, Costa do Sol, Cristo Redentor, Ernesto Geisel, Grotão, Gramame, Jardim Cidade Universitária, Jardim São Paulo, João Paulo Segundo, Mangabeira, Muçumagro e Jardim Botânico Benjamim Maranhão. Ao longo de seu percurso de aproximadamente $10 \mathrm{~km}$, o rio obedece ao sentido Oeste/ Leste, até desaguar no Oceano Atlântico na Praia do Sol onde se forma ecossistema estuarino.

\section{Localização da área e dos pontos de amostragens}

Foram adotados pontos de amostragem na bacia do rio Cuiá, sendo os pontos 1 e 2 correspondentes à zona de mistura provenientes dos lançamentos dos efluentes do sistema de tratamento de esgoto de Mangabeira (ETE-Mangabeira) no rio Cuiá, o ponto 3 à jusante e o ponto 4 à montante do ponto de lançamento da ETE-Mangabeira, como mostrado na Figura 1 e Tabela 1. 
Figura 1: Mapa de Localização da área e dos pontos de coletas do rio Cuiá.

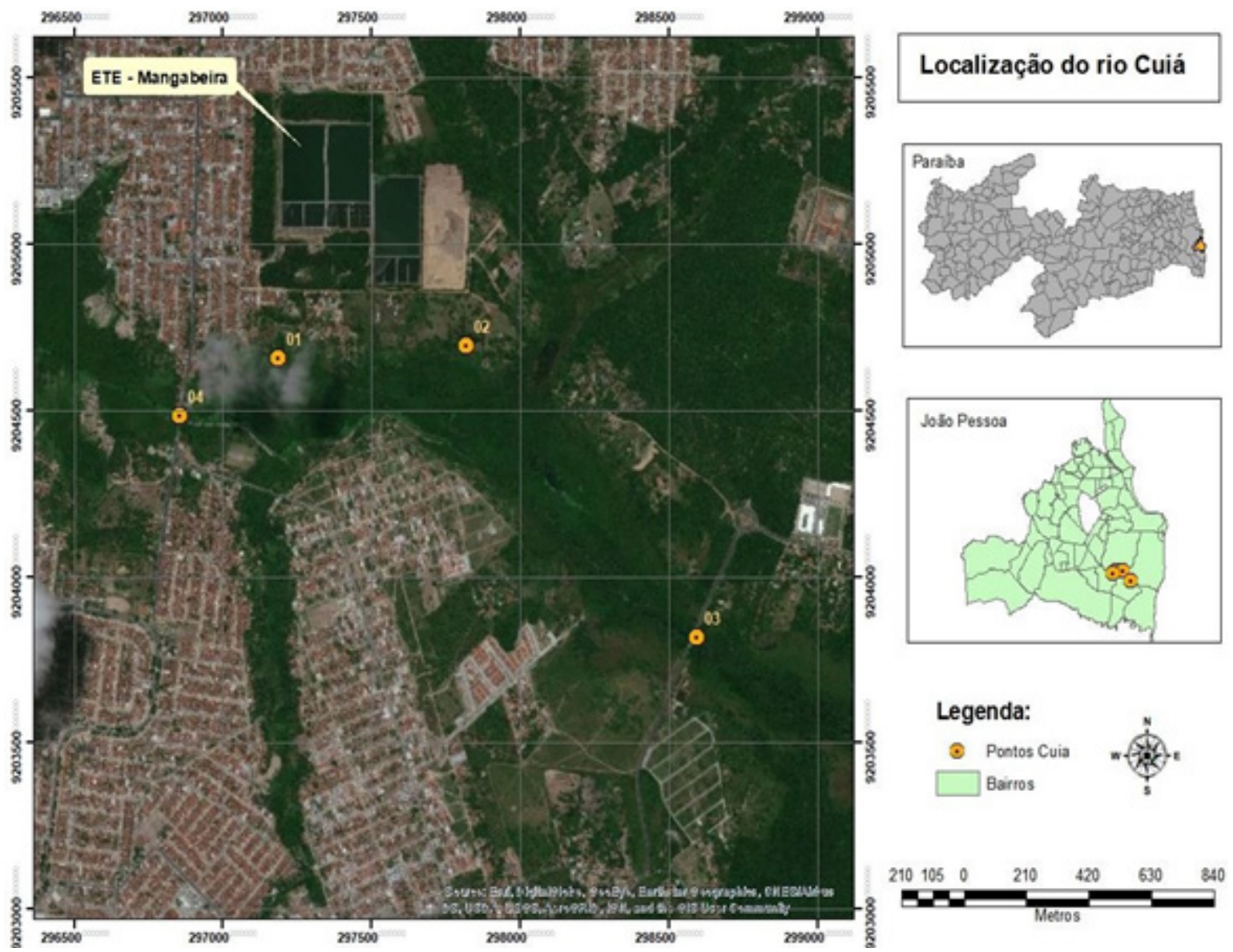

Fonte: os autores

Como observado na Figura 1, o ponto localizado a jusante da ETE-Mangabeira (ponto

3) está situado a margem da Rodovia Estadual PB008 e o ponto à montante desse sistema (ponto 4) está situado na divisa entre os bairros de Valentina Figueiredo e Mangabeira, bem como dois pontos localizados na zona de mistura (pontos 1 e 2), situados no encontro do lançamento do efluente da ETE-Mangabeira com o rio, identificados por círculos em cor laranja e representados pelas coordenadas UTM na Tabela 1.

\section{Tabela 1: Georreferenciamento dos pontos de coleta}

\begin{tabular}{|c|c|}
\hline *Pontos de Coleta & Coordenadas UTM \\
\hline Ponto 1 & $\begin{array}{c}7^{\circ} 11^{\prime} .30^{\prime \prime} \mathrm{S} \\
34^{\circ} 50^{\prime} 12.1 \text { " }\end{array}$ \\
\hline Ponto 2 & $\begin{array}{c}7^{\circ} 11^{\prime} 28.8^{\prime \prime} \mathrm{S} \\
34^{\circ} 49^{\prime} 51.4^{\prime \prime} \mathrm{O}\end{array}$ \\
\hline Ponto 3 & $\begin{array}{c}7^{\circ} 11^{\prime} 57.5 ” \mathrm{~S} \\
34^{\circ} 49^{\prime} 26.3 \text { "O }\end{array}$ \\
\hline Ponto 4 & $\begin{array}{c}7^{\circ} 11^{\prime} 35.6 ” \mathrm{~S} \\
34^{\circ} 50^{\prime} 22.9^{\prime \prime O}\end{array}$ \\
\hline
\end{tabular}

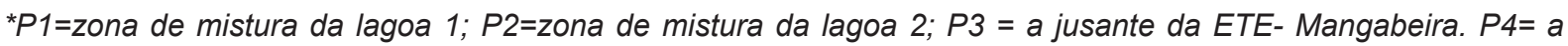
montante da ETE-Mangabeira; 
O período de obtenção das amostras ocorreu entre os anos de 2017 e 2018 e foram realizadas três campanhas amostrais, a primeira, em agosto de 2017, a segunda, em novembro do mesmo ano e a terceira, em maio de 2018. Em função da logística, foi padronizado o horário das coletas das amostras entre 8 e 9 horas da manhã.

As amostras foram coletadas, em duplicatas, levadas imediatamente ao laboratório e analisados os parâmetros, a saber, $\mathrm{pH}$, condutividade elétrica $(\mathrm{CE})$, cor, turbidez, temperatura, cloreto, dureza total, oxigênio dissolvido (OD) e nitrito, de acordo com o procedimento apresentado no Standard methods for the examination of water and wastewater (APHA, 2005).

\section{Uso e ocupação do solo}

Resultante da expansão urbana e das ações antrópicas, os recursos hídricos da bacia do rio Cuiá vêm sofrendo uma intensa e contínua degradação. Esta contestação foi observada através de um estudo de uso e ocupação do solo realizado ao longo do curso do rio (alto, médio e baixo) através de uma visita à bacia hidrográfica do rio Cuiá, no período que antecedeu as coletas de amostras de água. Além de seu espaço ocupado por diversos conjuntos residências, foi possível perceber a presença de vários criatórios de animais, bem como o lançamento de efluentes e de resíduos sólidos, como mostrado na Figura 2.

\section{Figura2- Possíveis aportes de contaminantes: (a) cavalaria (b) pocilga (c) resíduos sólidos (d) lançamento de efluente da ETE-Mangabeira}

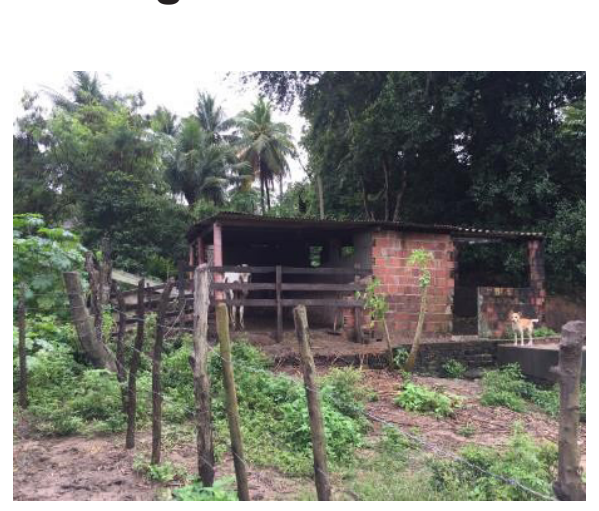

(a)

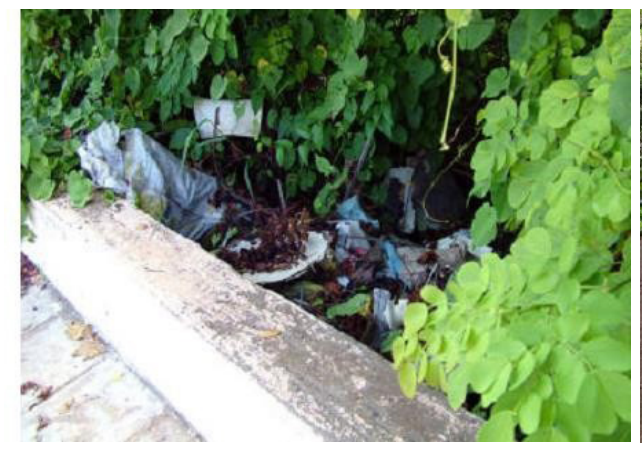

(c)

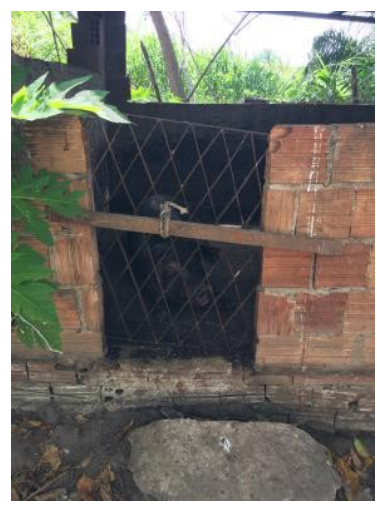

(b)

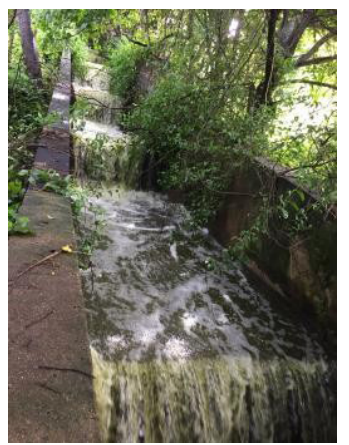

(d)

Fonte: os autores 


\section{4 Índice de Precipitação Pluviométrico}

O índice de precipitação pluviométrica referente ao período de realização do estudo (agosto de 2017 a julho de 2018) está apresentado na Figura 3.

\section{Figura 3. Índice de precipitação pluviométrica médio mensal no período de agosto de 2017 à julho de 2018}

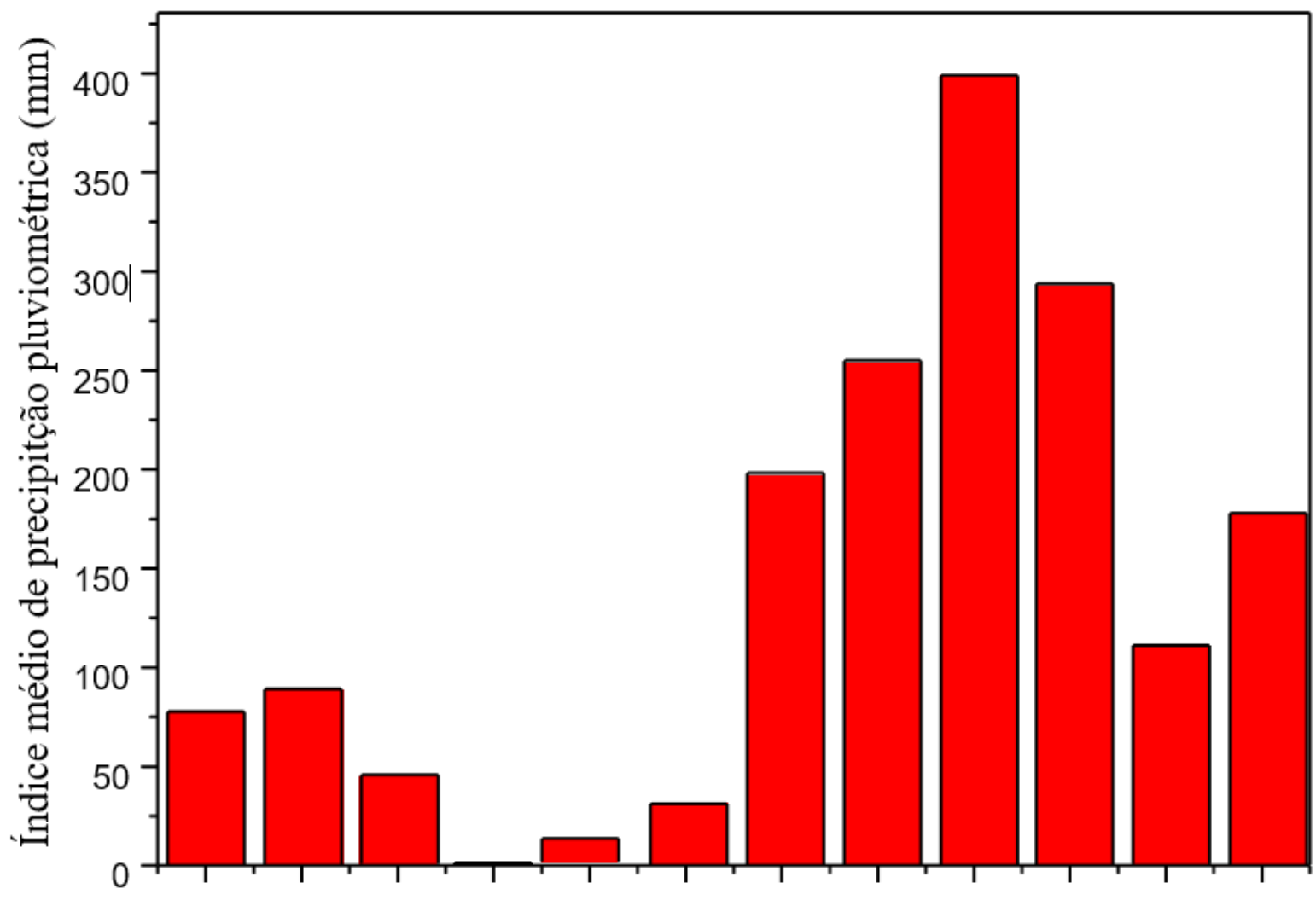

Ago/17 Set/17 Out/17 Nov/17 Dez/17 Jan/18 Fev/18 Mar/18 Abr/18 Mai/18 Jun/18 Jul/18

Fonte: AESA ( 2018)

\subsection{Análise dos dados}

O conjunto de dados (as matrizes) foi submetido ao auto-escalonamento, visto que ele é um método estatístico que visa eliminar a presença de desvios provenientes da diferença de unidades ou da diferença de ordem de grandeza dos dados que compõem a matriz. Ao realizar este processo, os dados não alteram seu padrão de variação, mas passam a ter uma variabilidade mais concisa e conhecida.

Os modelos de análise de componente principal (PCA) do conjunto de dados foram construídos empregando o método Full Cross validation, leave-one-out. Já os dendrogramas da Análise de Agrupamento Hierárquica (HCA) foram construídos usando o método Ward's e a distância Chebycher, dos dados brutos. 


\section{Resultados e Discussão}

\section{Análise de HCA}

A partir para a análise conjunta dos dados e observando o dendrograma, verifica-se que no estudo que é possível obter um padrão para o agrupamento das amostras, como pode ser observado na Figura 4.

\section{Figura 4. Dendrograma das amostras coletadas no período de agosto de 2017 a maio de 2018.}

Método Ward's

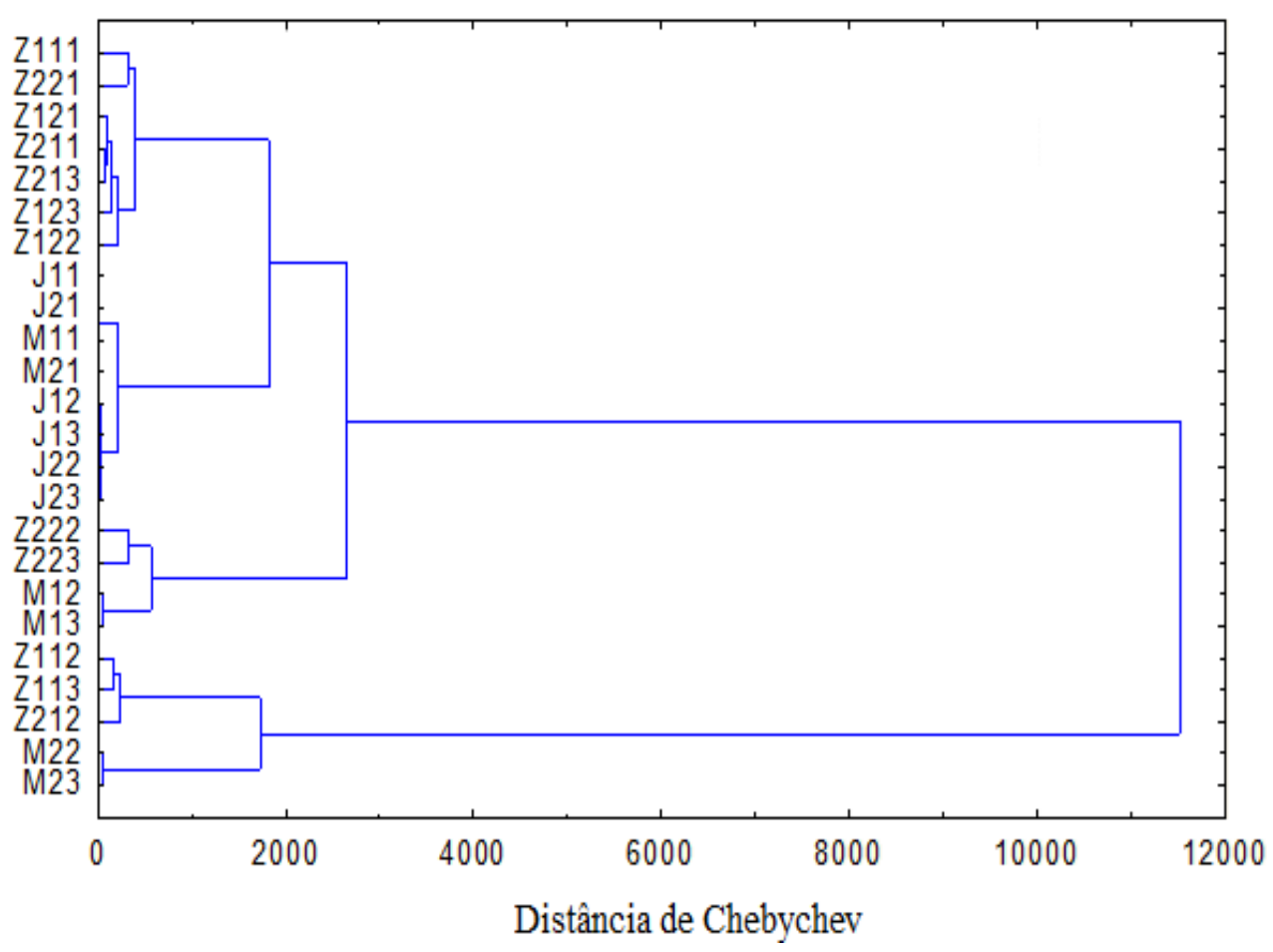

*Z111 e Z121==zona de mistura da lagoa 1, replicatas 1 e 2, $1^{a}$ coleta; *Z112 e Z122=zona de mistura da lagoa 1, replicatas 1 e 2, $2^{a}$ coleta; 'Z113 e Z123= =zona de mistura da lagoa 1, replicatas 1 e 2, $3^{a}$ coleta; *Z211 e Z221= =zona de mistura da lagoa 2, replicatas 1 e 2, 1ª coleta; 'Z212 e Z222=zona de mistura da lagoa 2, replicatas 1 e 2, $2^{a}$ coleta; "Z213 e Z223= =zona de mistura da lagoa 2, replicatas 1 e 2, 3a coleta; *M11 e M21 = a montante da zona de mistura das duas lagoas, replicata 1 e 2 e $1^{a}$ coleta; *M12 e M22 = a montante da zona de mistura das duas lagoas, replicatas 1 e 2 e $2^{a}$ coleta; M13 e M23 = a montante da zona de mistura das duas lagoas, replicatas 1 e 2 e $3^{a}$ coleta; *J11 e J21 = a jusante da zona de mistura das duas lagoas, replicatas 1 e 2 e $1^{a}$ coleta; * $J 12$ e J22 = a jusante da zona de mistura das duas lagoas, replicatas 1 e 2 e $2^{a}$ coleta; * $\mathrm{J} 13$ e J23 = a jusante da zona de mistura das duas lagoas, replicatas 1 e 2 e $3^{a}$ coleta.

O dendrograma da Figura 4 mostra que não foi possível observar a influência da sazonalidade (temporal), contudo foi favorável à análise espacial, visto que ocorre a segregação de um grupamento contendo quase que a totalidade das amostras da zona de mistura $(Z)$ e de mais dois ou três grupos, contendo amostras dos pontos a jusante $(\mathrm{J})$ e a montante $(\mathrm{M})$, com ou sem presença de amostras da zona de mistura. Essa segregação bem definida mostra uma qualidade da água da zona de mistura diferente dos pontos a montante e a jusante, não são condizentes com o comportamento esperado para o Rio Cuiá. 


\section{Análise de PCA}

Os modelos de PCA revelam que PC1 explica 51\% e PC2 22\% da variância dos dados.

Os resultados de PCA das análises temporais indicam um padrão de formação de grupo evidente, o que sugere que esse tipo de análise, utilizando essa técnica, é o ideal, uma vez que amostras de diferentes épocas do ano podem apresentar características muito diferentes.

As análises espaciais evidenciaram, em maior ou menor grau, a separação das amostras coletadas dos pontos a montante $(\mathrm{M})$ e a jusante $(\mathrm{J})$ das amostras coletadas dos pontos da zona de mistura (Z1 e Z2), indicando que esta separação está diretamente ligada à localização dos pontos de coleta. Esse comportamento foi observado em PC1 (Figura 5) no momento em que as amostras representadas por retângulos em vermelho ( $\mathrm{M} \mathrm{e} \mathrm{J}$ ) encontram-se com altos escores negativos em PC1 e as representadas em círculos azuis ( $\mathrm{Z} 1$ e $\mathrm{Z} 2)$, altos escores positivos nessa mesma componente.

Tais resultados indicam que PC1 está relacionado com a qualidade da água do rio, uma vez que EC, CT, turbidez, cor e CE tem pesos bastante positivos nesta componente (Figura 6) sendo as variáveis $\mathrm{EC} \mathrm{e} \mathrm{CT} \mathrm{responsáveis} \mathrm{por} \mathrm{contaminação} \mathrm{bacteriológica} \mathrm{e} \mathrm{pH}$, Cor, turbidez e $\mathrm{CE}$, associados ao lançamento de efluentes provenientes das lagoas facultativas e as principais variáveis responsáveis por esta separação. Esse comportamento já era esperado, uma vez que as amostras da zona de mistura são provenientes do efluente da ETE-Mangabeira, na qual possivelmente nesse ponto ainda não houve tempo para a degradação por autodepuração. Estes resultados corroboram os apresentados por HCA, apresentados na Figura 4.

\section{Figura 5. Gráfico dos escores de PC1XPC2 em função dos pontos de coletas. A montante e a jusante ( ) ) e zona de mistura ( • )}

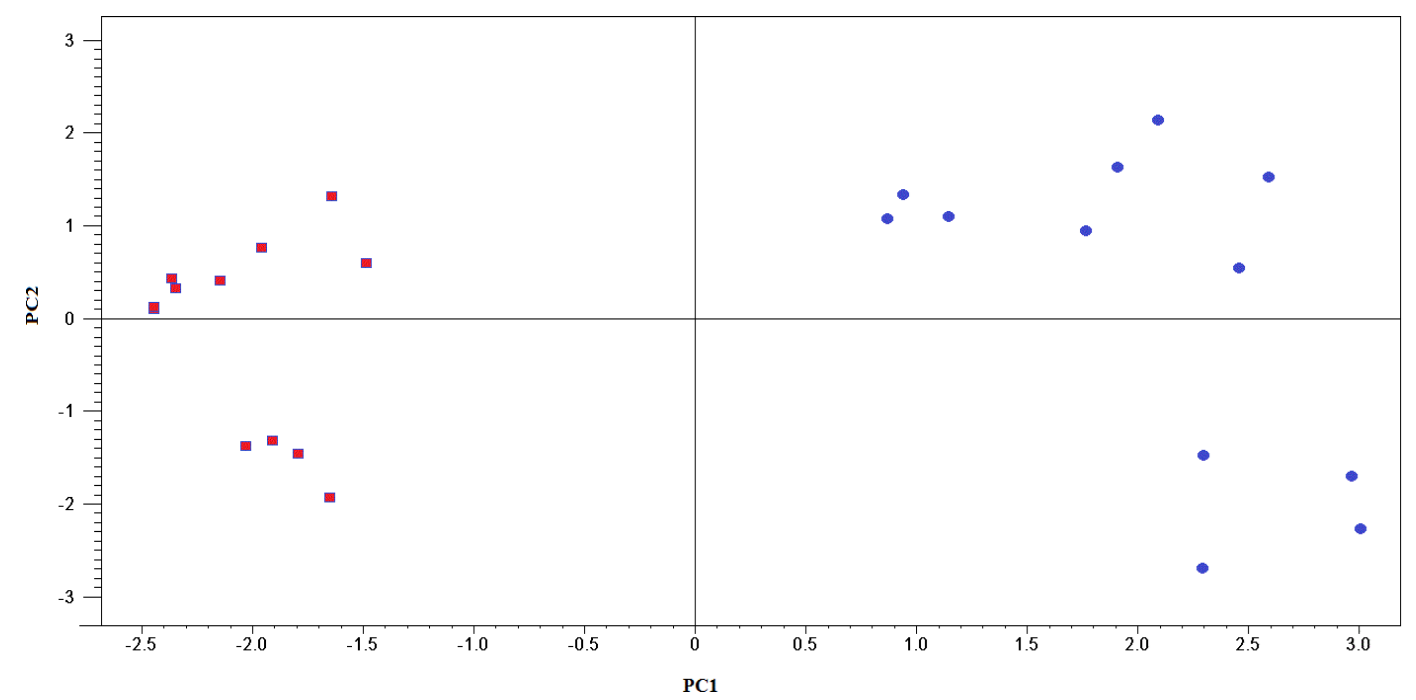

O gráfico dos pesos de PCA estão mostrados na Figura 6. 
Figura 6. Gráfico dos pesos de PC1 X PC2

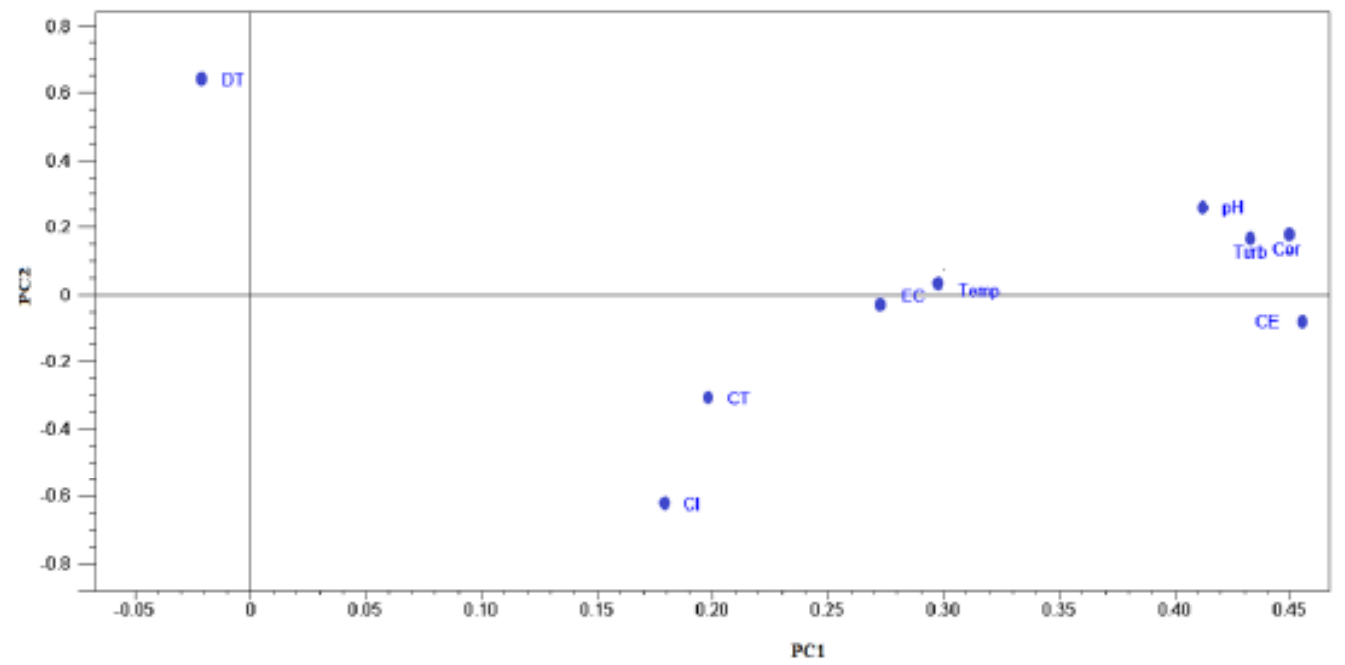

$\mathrm{O}$ gráfico da Figura 6 mostra que as variáveis cor aparente, $\mathrm{pH}$, turbidez e $\mathrm{CE}$ estão correlacionadas, visto que elas apresentam valores de escores próximos entre si, tanto em PC1 quanto em PC2. Esses resultados indicam que é possível reduzir a dimensionalidade inerente do sistema para 6 variáveis.

Os resultados de PCA, em função do período de coleta estão mostrados na Figura 7.

Figura 7- Gráfico dos escores de PC1XPC2 em função do período de coleta: $1^{\text {a }}$ coleta, agosto/2017 ( ( ) , $2^{\mathrm{a}}$ coleta, novembro/2017 $(\cdot)$ e $3^{a}$ coleta, maio/2018 (•)

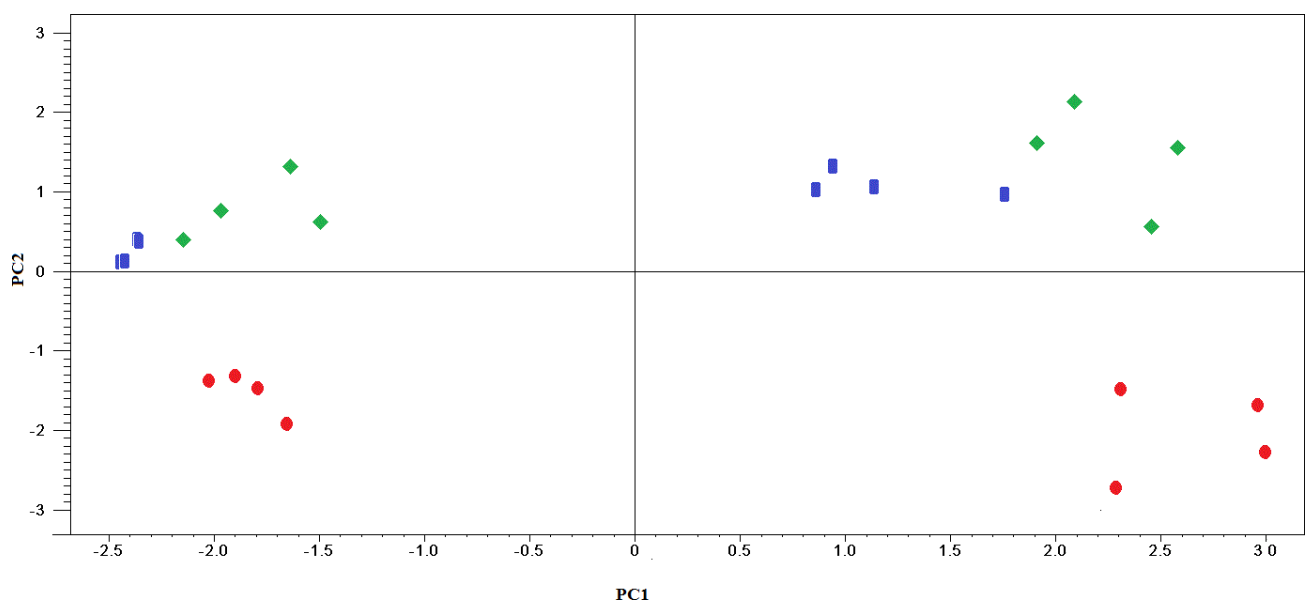

A PC2 (Figura 7) está associada aos fenômenos da sazonalidade que ocorrem devido às três campanhas amostrais realizadas em agosto/2017 e maio/2018, referentes ao período de chuva, e das amostras de novembro/2017, do período seco (Figura 4). Tais fenômenos evidenciaram uma clara separação entre essas amostras. Este comportamento foi observado na Figura 7, quando as amostras da segunda coleta $(\bullet)$ apresentam altos pesos negativos em PC2 enquanto que a maioria das amostras da primeira $(\bullet)$ e terceira $(\bullet)$ coletas apresentam altos pesos nessa componente. 


\section{Conclusões}

Os resultados obtidos através da análise exploratória multivariada, por meio das análises de agrupamento hierárquico (HCA) e de componentes principais (PCA), corroboram a hipótese de que é possível sua utilização para verificação de padrões comportamentais, bem como elucidarem a influência temporal e espacial causadas pelas variáveis a serem analisadas.

As análises realizadas nas variáveis mostraram de que forma se encontram interligadas, permitindo excluir do modelo as variáveis não representativas e selecionar quais são as mais representativas a serem utilizadas na criação de modelos ambientais, a fim de assegurar a manutenção de parâmetros mínimos de qualidade de água e um monitoramento mais efetivo dessa matriz ambiental.

A análise espacial mostrou-se favorável do ponto de vista de HCA e PCA, contudo a análise temporal apresentou resultados interessantes apenas sob a ótica da análise de componentes principais. Os resultados mostram que foi possível reduzir a dimensionalidade inerente do sistema, visto que as variáveis $\mathrm{pH}$, cor, turbidez e condutividade elétrica estão fortemente correlacionadas, podendo três dessas não serem incluídas do modelo e, consequentemente, do programa de monitoramento. Tais resultados indicam que a variação da qualidade da água do rio Cuiá está intrinsecamente ligada ao lançamento do efluente da ETE-Mangabeira, visto que ambas as técnicas evidenciaram a segregação de dois dos pontos (pontos 1 e 3) com relação aos demais (pontos 2 e 4) o que sugere que a criação de modelos espaciais também é possível.

As técnicas de análises exploratórias empregadas mostraram-se satisfatórias, tendo em vista que tornaram possível a execução da proposta inicial, bem como sinalizaram que podem ser utilizadas para conjuntos de dados semelhantes. Assim, podemos concluir que as ferramentas estatísticas demonstraram-se importantes para o entendimento do comportamento do sistema estudado, o que possibilita subsidiar a tomada de decisão dos gestores da bacia hidrográfica do rio Cuiá frente às ameaças de contaminação desse ecossistema.

\section{Referências}

AESA-Agência Executiva de Gestão das Águas da Paraiba. Disponível em: http://www. aesa.pb.gov.br/aesa-website/meteorologia-chuvas. Acesso em: 27 de setembro de 2018.

APHA-AMERICAN PUBLIC HEALTH ASSOCIATION et al. Standard methods for the examination of water and wastewater. 21th ed. Washington, 2005. 1274 p.

\footnotetext{
BRASIL. Conselho Nacional do Meio Ambiente. Resolução CONAMA n. 357, de 17 de março de 2005. Dispõe sobre a classificação dos corpos de água e diretrizes ambientais para o seu enquadramento, bem como estabelece as condições e padrões de lançamento de efluentes, e dá outras providências. Brasília, Diário Oficial [da] União, n. 53, 18 mar. 2005, p. 58-63.
} 
BRASIL. Ministério da Saúde. Portaria n ${ }^{\circ}$ 2.914, de 12 de dezembro de 2011. Dispõe sobre os procedimentos de controle e de vigilância da qualidade da água para consumo humano e seu padrão de potabilidade. Diário Oficial da República Federativa do Brasil, Poder Executivo, Brasília, DF, 14 dez. 2011. Seção 1, p. 39-42. Disponível em: http:// bvsms.saude.gov.br/bvs/saudelegis/gm/2011/prt2914_12_12_2011.html. Acesso em: 03 de setembro de 2018.

BRASIL. Presidência da República. Lei No 9.433 de 8 de janeiro de 1997. Institui a Política Nacional de Recursos Hídricos, cria o Sistema Nacional de Gerenciamento de Recursos Hídricos. Disponível em: http://www3.ana.gov.br/portal/ANA/ todos-os-documentos-do- portal/documentos-sre/alocacao-de-agua/oficina-escassezhidrica/legislacao-sobre-escassez- hidrica/uniao/lei-no-9433-1997-pnrh/view. Acesso em 08 de setembro de 2018.

BUSSAD, W. O.; MIAZAKI, E. S.; ANDRADE, D. F. Introdução à análise de agrupamentos. In: SIMPÓSIO NACIONAL DE PROBABILIDADE E ESTATÍSTICA, 9., São Paulo, 1990. 105 p.

CALIJURI, M. C. (1988). Respostas fisioecológicas da comunidade fitoplanctônica e fatores ecológicos em ecossistemas com diferentes estágios de eutrofização. 293p. Tese (Doutorado). Ciências da Engenharia Ambiental, Escola de Engenharia de São Carlos, Universidade de São Paulo, São Carlos. 1988.

DETONI, T. L.; DONDONI, P. C. A Escassez da água: um olhar global sobre a sustentabilidade e a consciência acadêmica. Rev. Ciênc. Admin., v. 14, n. 2 , p. 191-204, 2008.

FAO - Food and Agriculture Organization of the United Nations. Towards a water and food secure future: critical perspectives for policy-makers. Rome: FAO, 2015.76p.

FERREIRA, M. M. C; ANTUNES, A. M.; MELGO, M. S.; VOLPE, P. L. O. Quimiometria I:

calibração multivariada, um tutorial. Quim. Nova, v.22, p.724-731, 1999.

GIRÃO, E. G.; ANDRADE, E. M.; ROSA, M. F.; ARAÚJO, L. F. P.; MEIRELES, A. C.M.

Seleção dos indicadores da qualidade de água no Rio Jaibaras pelo emprego da análise da componente principal. Revista Ciência Agronômica, v.38, n.1, p.17-24, 2007.

HAIR JUNIOR, J. F.; ANDERSON, R. E.; TATHAM, R. L.; BLACK, W. C. Análise multivariada de dados. Tradução de Adonai Schlup Sant'Anna e Anselmo Chaves Neto, 5. ed. Porto Alegre: Bookman, 2005.593p. 
HASWELL, S.,J.; WALMSLEY, A. D. Multivariate data visualization methods based on multi-elemental analysis of wines and coffees using total refletion $\mathrm{X}$-ray fluorescence analysis. J. Anal. At. Spectrom., v.13, p.131-134, 1998.

HIDALGO, P. Diagnóstico socioeconômico. João Pessoa: PRM/METROPLAN/ AGP-RS.1992.98p. Curso sobre Manejo Conservacionista de Bacias Hidrográficas. 1992.

JONG,J.; ROOY,P.T.J.C.; HOSPER, S.H.(1995). Living with water: at the crossroads of change. Water Science Technology. Institute for Inland Water Management and WasteWater Treatment, The Netherlands, n. 8, p.393-400.

LEMOS, W. E. D. Monitoramento e gestão da qualidade da água em reservatórios incorporando processos hidrodinâmicos e climáticos de regiões tropicais semiáridas. 2011. 164 f. Dissertação (Mestrado) - Universidade Federal do Ceará, Fortaleza, 2011. Disponível em: < http://www.repositorio.ufc.br/handle/riufc/575>. Acesso em $27 \mathrm{de}$ setembro de 2018.

MENDIGUCHÍA, C.; MORENO, C.; GALINDO-RIANO, M.D.; GARCÍAVARGAS, M.

Using chemometric tools to assess antropogenic effects in river water a case study: Guadalquivir river (Spain). Analytica Chimica Acta, Amsterdam, v.515, p.143-9, abr., 2004.

SENA M. M.; POPPI, R. J.; FRIGHETTO, R. T. S.; VALARINI, P. J. Avaliação do uso de

métodos quimiométricos em análise de solos. Quim. Nova, v.23, p.547-556, 2000.

SINGH, K.P.; MALIK,A.; MOHAN, D.; SINHA, S.Multivariate statistical techniques for the evaluation of spatial and temporal variations in water quality of Gomti River (India) - a case sudy. Water Research, n. 38, p. 3980-3992, 2004.

SOUSA, A. C. Avaliação do funcionamento de uma estação de tratamento de esgoto doméstico e desenvolvimento de um novo método para determinação de DQO usando espectrometria NIR e quimiometria. 2007. 103 f. Tese (Doutorado em Química) Universidade Federal da Paraíba, João Pessoa, 2007.

SUNDARAY, S. K.; PANDA, U. C.; NAYAK, B. B.; BHATTA, D. Multivariate statistical techniques for the evaluation of spatial and temporal variations in water quality of the Mahanadi river-estuarine system (India)—a case study. Environmental Geochemistry Health, v.28, p.317-330, 2006.

TELLES, D. D. A água e o ambiente. In: . Ciclo ambiental da água: da chuva à gestão. São Paulo: Blucher, 2013. 
TOLEDO, L. G.; NICOLELLA, G. Índice de qualidade de água em microbacia sob uso agrícola e urbano. Scientia Agrícola, Piracicaba, v.59, p.181-6, 2002.

VEGA, M.; PARDO, R.; BARRADO, E.; DEBAÂ, L. Assessment of seasonal and polluting effects on the quality of river water by exploratory data analysis. Water Research, v.32, p.3581-3592, 1998.

VIDAL, M.; LÓPEZ, A.; SANTOALLA, M.C.; VALLES, V. Factor analysis for the study of water resources contamination due to the use of livestock slurries as fertilizer. Agricultural Water Management, v.45, p.1-15, 2000. 